\title{
A 5-year follow-up study of Alfredson's heel-drop exercise programme in chronic midportion Achilles tendinopathy
}

\author{
A van der Plas, ${ }^{1} \mathrm{~S}$ de Jonge, ${ }^{1,2} \mathrm{R} \mathrm{J}$ de Vos, ${ }^{1,2} \mathrm{H} \mathrm{J} \mathrm{L}$ van der Heide, ${ }^{3} \mathrm{~J}$ A N Verhaar, ${ }^{2}$ \\ A Weir, ${ }^{1} \mathrm{~J} \mathrm{LTOl}^{1}$
}

1 Sports Medicine Department, The Hague Medical Centre, Leidschendam, The Netherlands ${ }^{2}$ Department of Orthopaedics, Erasmus Medical Centre, Rotterdam, The Netherlands ${ }^{3}$ Department of Orthopaedics, Leiden University Medical Centre, Leiden, The Netherlands

\section{Correspondence to} Dr J L Tol, Sports

Medicine Department, The Hague Medical Centre Antoniushove, P0 Box 411, Burgemeester Banninglaan 1, 2260 AK Leidschendam,

The Netherlands:

h.tol@mchaaglanden.nl

Received 16 March 2011 Accepted 8 September 2011 Published Online First 10 November 2011

\section{(6) UNLOCKED}

This paper is freely available online under the BMJ Journals unlocked scheme, see http:// bjsm.bmj.com/info/unlocked.dtl

\begin{abstract}
Background Eccentric exercises have the most evidence in conservative treatment of midportion Achilles tendinopathy. Although short-term studies show significant improvement, little is known of the long-term ( $>3$ years) results.
\end{abstract}

Aim To evaluate the 5 -year outcome of patients with chronic midportion Achilles tendinopathy treated with the classical Alfredson's heel-drop exercise programme.

Study design Part of a 5-year follow-up of a previously conducted randomised controlled trial.

Methods 58 patients (70 tendons) were approached 5 years after the start of the heel-drop exercise programme according to Alfredson. At baseline and at 5-year follow-up, the validated Victorian Institute of Sports Assessment-Achilles (VISA-A) questionnaire score, pain status, alternative treatments received and ultrasonographic neovascularisation score were recorded.

Results In 46 patients (58 tendons), the VISA-A score significantly increased from 49.2 at baseline to 83.6 after 5 years $(p<0.001)$ and from the 1-year to 5 -year follow-up from 75.0 to $83.4(p<0.01) .39 .7 \%$ of the patients were completely pain-free at follow-up and $48.3 \%$ had received one or more alternative treatments. The sagittal tendon thickness decreased from $8.05 \mathrm{~mm}$ (SD 2.1) at baseline to $7.50 \mathrm{~mm}$ (SD 1.6) at the 5-year follow-up ( $p=0.051$ ).

Conclusion At 5-year follow-up, a significant increase of VISA-A score can be expected. After the 3-month Alfredson's heel-drop exercise programme, almost half of the patients had received other therapies. Although improvement of symptoms can be expected at long term, mild pain may remain.

\section{INTRODUCTION}

There are many conservative treatment options for chronic Achilles tendinopathy. Systematic reviews showed that the heavy load eccentric exercise programme has the most evidence of effectiveness in the treatment of chronic midportion Achilles tendinopathy. ${ }^{1-4}$ The reviews demonstrated promising results on pain. However, there is limited evidence to show that eccentric exercises are more effective than other treatments in the management of tendinopathies. The exact mechanism involved in the eccentric exercises is unclear and there is an ongoing debate on the optimal load, frequency and duration. ${ }^{5}{ }^{6}$ It is suggested that eccentric training might stimulate remodelling and tissue repair in tendons, the so-called therapeutic mechano transduction. ${ }^{7}$ The classical heel-drop exercise programme first described by Alfredson et al is time-consuming (180 repetitions per day for 12 weeks). The majority of the published studies have a maximum follow-up of 1 year, and there is a lack of evidence about the long-term ( $>3$ years) outcome. ${ }^{9-13}$ Three studies evaluated the long-term ( $>3$ years) results of the eccentric exercises in chronic midportion Achilles tendinopathy. ${ }^{14-16}$ Only one study used the validated Victorian Institute of Sports Assessment-Achilles (VISA-A) questionnaire, but in that study the exercise regimen differed from that described by Alfredson et al ${ }^{6}$ The VISA-A questionnaire is a validated score that measures pain and physical activity of the Achilles tendon. ${ }^{17}$ The test is developed to create an index of severity of Achilles tendinopathy.

So far, no long-term VISA-A score results (>3 years) are known of the widely used heel-drop programme according to Alfredson. We evaluated the long-term clinical outcome of the heel-drop programme as described by Alfredson with the VISA-A score as the primary outcome measure. We also evaluated the ultrasonographic improvement 5 years after conservative therapy, alternative treatments received, pain status and patient satisfaction.

\section{METHODS}

This study is the 5-year follow-up of an earlier published randomised controlled trial (RCT). ${ }^{13}$ The RCT evaluated the effect of the addition of a night splint to eccentric exercises in patients with chronic Achilles tendinopathy. At baseline, 58 patients (70 tendons) with chronic midportion Achilles tendinopathy were included. Inclusion criteria at baseline were the presence of symptoms for more than 2 months and participation in sporting activities. All patients in the trial preformed the heel-drop programme as described by Alfredson et al. ${ }^{8}$ Half of the patients additionally received a night splint. The night splint had no significant influence on the VISA-A score at the 3-month and 1-year follow-up; all patients were therefore merged into one group for the 5-year follow-up..$^{13} 18$ After completion of the heel-drop programme, patients were free to choose additional treatment(s); no instructions or recommendations were given to the patients. 
The study protocol was approved by the Medical Ethics Committee of the Hospital (MEC05-21) and informed consent was given.

\section{Follow-up}

All patients were approached by telephone for the 5-year follow-up. At the outpatient clinic, each patient completed the VISA-A score questionnaire and received an ultrasound examination by a single researcher. The VISA-A score was completed with minimal researcher assistance. Secondary outcome scores were obtained using a standardised questionnaire: (1) pain status (pain-free, not pain-free), (2) condition of the uninvolved tendon (affected, not affected), (3) subjective patient satisfaction towards the current status (categorised in poor, moderate, good, excellent), (4) received alternative treatments after the heel-drop programme and, (5) continuation of the eccentric exercises (continued, stopped after the heel-drop programme).

\section{Ultrasound examination}

For ultrasound examination, patients were asked to lie prone with their feet hanging over the edge of the table. During the examination, both Achilles tendons were evaluated in the sagittal plane for thickness and neovascularisation according to the Öhberg scoring system. This score was evaluated as 0 (no vessels visible), $1+$ (one vessel mostly in the anterior part), $2+$ (one or two vessels throughout the tendon), $3+$ (three vessels throughout the tendon) and $4+$ (more than three large vessels throughout the tendon). Two different power Doppler machines were used to examine the tendons: the MyLab25Gold (Esaote Piemedical, Maastricht, The Netherlands) and the BK Pro Focus (BK Medical, Herlev, Denmark). Both power Doppler machines used a frequency of $12 \mathrm{MHz}, 0.5 \mathrm{PRF}$ (pulse repetition frequency). The colour gain setting was optimised for low flow; putting the setting just below that level produces random noise, varying from $66 \%$ to $79 \% .{ }^{19}$

\section{Statistical analysis}

Differences in VISA-A score and tendon thickness between baseline and follow-up were evaluated using a sample t test. Differences between two categorical variables were evaluated using a $\chi^{2}$ test. Dependency analysis of the VISA-A score was performed using a non-repetitive analysis of covariance test. Statistical Package for the Social Sciences software (SPSS 17.0) was used. A P value of less than 0.05 was considered to be statistically significant.

\section{RESULTS}

Attempts were made to contact all 58 patients (70 tendons). Six patients (six tendons) were not reachable for follow-up and five patients (five tendons) refused to participate (see Figure 1). Three out of these five patients reported having no or minimal complaints of the Achilles tendon; unfortunately, further details are unknown. The VISA-A score and questionnaire results were obtained from 47 patients (59 tendons): 12 bilateral and 35 unilateral. One patient (one tendon) was excluded from analysis due to a direct trauma to the Achilles tendon 2 days before the follow-up appointment, making the outcome non-representative. For general statistical analysis, 46 patients (58 tendons) were used. Five patients (six tendons) were not able to visit the hospital (no time or living abroad) but filled out the general questionnaire and the VISA-A score (see figure 1 for details). The ultrasonographic data consisted of information from 41 patients (52 tendons).

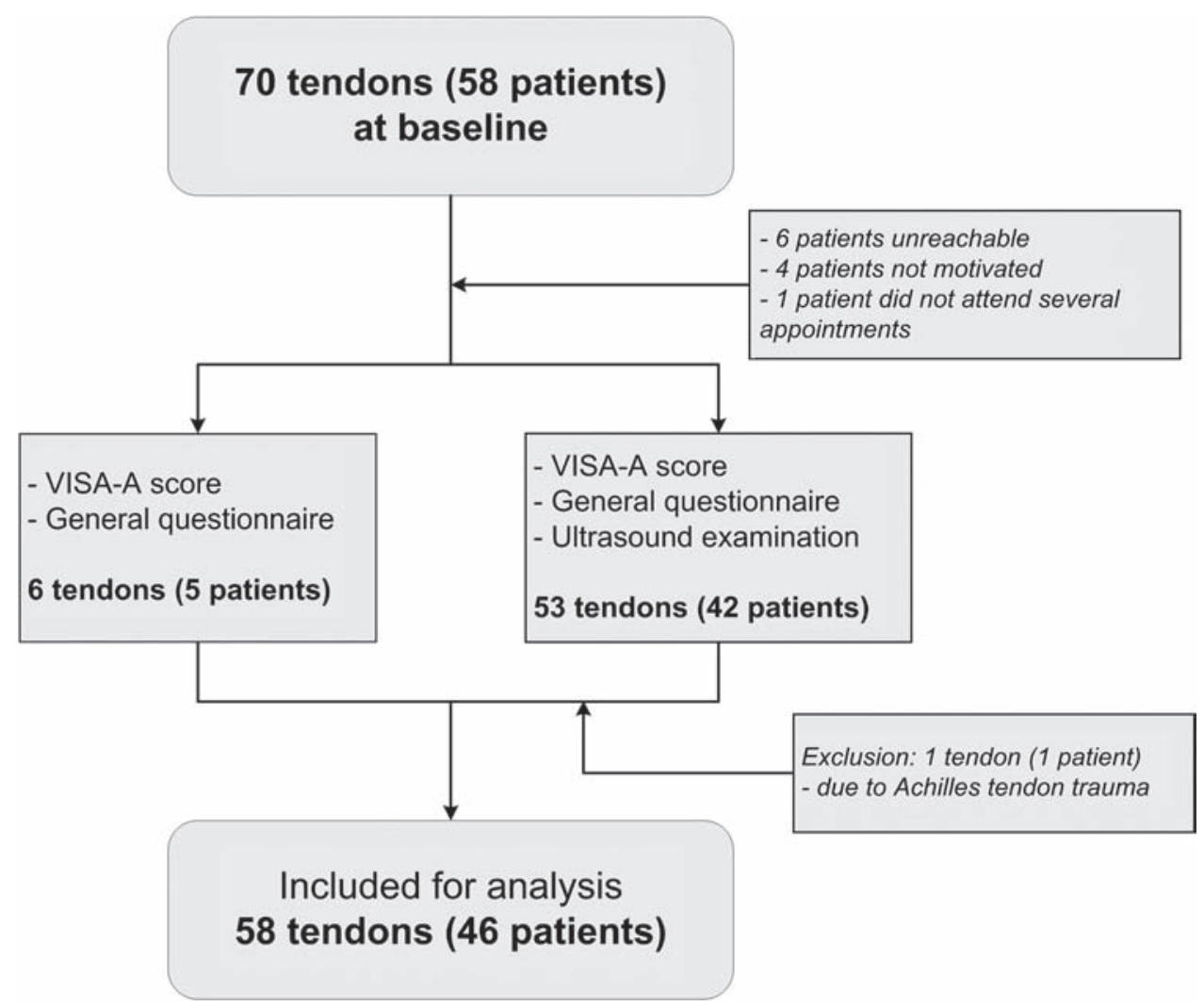

Figure 1 Flowchart of included patients. 
Table 1 Patient characteristics at 5-year follow-up

\begin{tabular}{ll}
\hline & $\mathbf{4 6}$ Patients (58 tendons) \\
\hline Mean age (years (range)) & $50.9(36-64)$ \\
Body mass index (kg/m² (range)) & $25.4(19.9-33.5)$ \\
Unilateral/bilateral & $34 / 12$ \\
No sports activity & 4 \\
Recreational sports & 35 \\
Competitive sports & 7 \\
\hline
\end{tabular}

At follow-up, the mean age was 50.9 years, with a mean body mass index of $25.4 \mathrm{~kg} / \mathrm{m}^{2}$ (see table 1 for details). Baseline specifics are described by de Vos et al. ${ }^{13}$

\section{Primary outcome: VISA-A score}

The VISA-A score $(n=58)$ improved significantly from 49.2 (SD 20.1) at baseline to 83.6 (SD 14.9) at the 5-year followup $(\mathrm{p}<0.001)$. The VISA-A score $(\mathrm{n}=55)$ improved significantly between the 1-year follow-up and the 5-year follow-up from 75.0 (SD 25.0) to 83.4 (SD 15.1) ( $p=0.006)$. See figure 2 for the trend line of the VISA-A score.

\section{Pain status}

Of the patients, $39.7 \%$ reported being completely pain-free at the 5 -year follow-up (mean VISA-A score 92.8, SD 8.8). The remaining $60.3 \%$ experienced some degree of pain, varying from only during extensive exercise to permanent pain (mean VISA-A score 77.5, SD 15.1). From the 34 patients with unilateral Achilles tendinopathy, 43.3\% developed some degree of pain in the contralateral Achilles tendon.

\section{Patient satisfaction}

Patient subjective satisfaction towards the current status was excellent in $8.6 \%$ (mean VISA-A score 89.0, SD 8.0), good in $41.4 \%$ (mean VISA-A score $84.6, \mathrm{SD} 16.3$ ), moderate in $31.0 \%$ (mean VISA-A score 84.4 , SD 15.2) and poor in $19.0 \%$ (mean VISA-A score 77.6, SD 13.5).

\section{Alternative treatments}

In total, $48.3 \%$ (22 patients) had received one or more alternative treatments, non-custom-made inlays (11 patients), surgery (6 patients), physiotherapy/massage/friction (6 patients), shockwave therapy (2 patients), immobilisation cast ( 2 patients), Polidocanol injection (1 patient), platelet-rich plasma injection (1 patient), glyceryl trinitrate patches (1 patient), acupuncture (1 patient), non-steroidal anti-inflammatory drugs (1 patient) and rest for 2 years ( 1 patient). Of the group of patients treated with an alternative treatment, $21.4 \%$ was completely painfree. In the group of patients who did not receive an alternative treatment, $56.7 \%$ was completely pain-free. After completing the 3 months heel-drop programme in the original study, $67.2 \%$ (31 patients) never performed the eccentric exercises again. No correlation in pain status was found between patients who continued the eccentric exercises and those who did not $(p=0.15)$.

\section{Ultrasonographic examination}

The sagittal thickness decreased from $8.1 \mathrm{~mm}$ (SD 2.1) at baseline to $7.5 \mathrm{~mm}(\mathrm{SD} \mathrm{1.6)}$ at the 5 -year follow-up ( $\mathrm{n}=50$, $\mathrm{p}=0.051$ ). At baseline, $58.8 \%$ had some degree of neovascularisation (Öhberg score 1 to $4+$ ). After 5 years, $47 \%$ showed

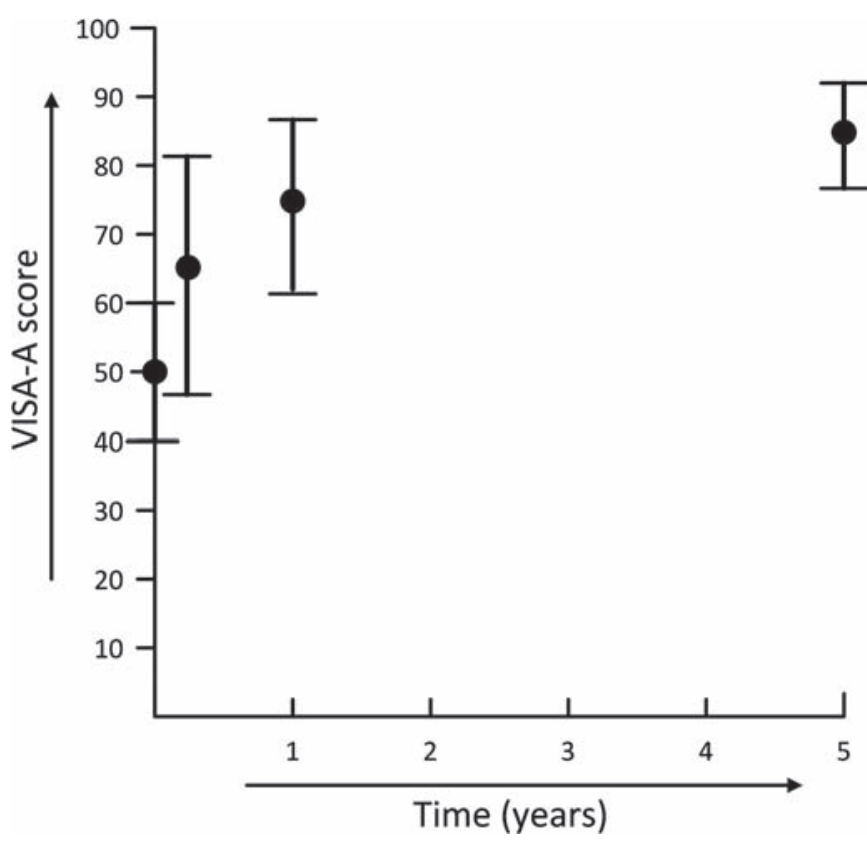

Figure 2 The VISA-A score in time.

neovascularisation during the ultrasonographic examination $(\mathrm{n}=51)$. There was no difference in increase in VISA-A score between the patients with neovessels at baseline (increase of 32.1 points, SD 22.1) and patients without neovessels at baseline (increase of 32.1 points, SD 23.7).

Fifty-four per cent of the patients without pain at followup had some degree of neovascularisation. No significant difference was found in tendon thickness at 5-year follow-up between the patients with and without symptoms $(p=0.59)$.

\section{Factors influencing the VISA-A score}

The VISA-A score at 5-year follow-up and the $\Delta$ VISA-A (= difference between baseline and the 5-year follow-up) score were not influenced by age $(p=0.61$ resp. $p=0.18)$, sex $(p=0.50$ resp. $p=0.39)$, body mass index $(p=0.83$ resp. $p=0.50)$, duration of symptoms at baseline $(p=0.11$ resp. $p=0.27)$, degree of neovascularisation at baseline $(p=0.53$ resp. $p=0.81)$ and sagittal tendon thickness at baseline $(p=0.43$ resp. $p=0.39)$.

\section{DISCUSSION}

This is the first study evaluating the 5 -years results of the Alfredson heel-drop programme with the validated VISA-A score. The VISA-A score increased significantly from 49.2 at baseline to 83.6 at the 5 -year follow-up and from the 1-year follow-up to 5-year follow-up from 75.0 to 83.4 ( $p<0.01$ ).

Until now the VISA-A score has only been used in one long-term follow-up study on eccentric exercises in Achilles tendinopathy. ${ }^{16}$ Silbernagel et al reported a VISA-A score of 90.5 at 5 -year follow-up, $65 \%$ of symptom-free patients and approximately $6 \%$ receiving alternative treatments. In their study, the training programme lasted from 12 weeks to 6 months and was performed under the supervision of a physiotherapist. Patients in the current study received very accurate instruction for the heel-drop programme and were instructed to do the exercises with pain. The compliance for the exercises was good/excellent in the majority of the patients $(>70 \%) .{ }^{13}$ The basic population of Silbernagel et al 
showed many similarities with our population, however, no competitive athletes were included in the study of Silbernagel et al. Maffulli et a ${ }^{20}$ suggested there could be a difference in response to eccentric training in the Scandinavian population. Several Scandinavian studies have demonstrated good/ excellent short-term results from the eccentric exercises in the treatment of chronic Achilles tendinopathy. ${ }^{9-12}$ In recent years, differing results have been found by non-Scandinavian studies showing only moderate short-term results. ${ }^{13} 21$ 22 The long-term Scandinavian studies evaluating eccentric exercises in chronic Achilles tendinopathy suggest a good long-term prognosis. ${ }^{14-16}$ Further studies will be needed to reveal if there is a genetic explanation for this difference. ${ }^{23}$

Two other studies have reported long-term effects of the heel-drop programme used in this study in patients with Achilles tendinopathy. ${ }^{14} 15$ However, neither of them used the validated VISA-A score. Ohberg et al showed that 22 of $25(88 \%)$ patients were satisfied with the treatment at a mean follow-up of 3.8 years (range 1.6-7.75). The second long-term follow-up study reported that $65 \%$ of the patients had no or mild pain after 4.2 years (range 29-58 months). ${ }^{14}$ In our study, $39.7 \%$ of the study population was completely pain-free at the 5-year follow-up. Almost half of our patients had received one or more alternative treatments. These secondary subjective outcomes suggest a discrepancy with the significant increase of the primary outcome score. For advise to patients it implies that a quantified improvement can be expected, but mild remaining pain symptoms may be present at long term.

Ultrasonographic evaluation demonstrated a comparable decrease in tendon thickness from $8.8 \mathrm{~mm}$ before the heel-drop programme to $7.6 \mathrm{~mm}$ at follow-up. However, the clinical relevance of this minor decrease is questionable. In recent years, a debate has arisen about the meaning and the significance of neovascularisation in Achilles tendons. Some studies have found a correlation between the patient's symptoms and the degree of neovascularisation, ${ }^{24-26}$ and others found that the presence of neovascularisation did not per se indicate a disease state. ${ }^{27-31}$ Also the circumstances during the examination, for example, activity just before the measurement, was not controlled and is unknown. This study found that the VISA-A score was not related to the degree of neovascularisation. Further research is needed to understand the relevance of neovascularisation in Achilles tendons.

This study has some limitations. The 11 dropouts might have influenced the outcome. In a best case scenario analysis where the dropouts have the best outcome score, $50 \%$ of the patients would be pain-free. However, the value of this non-validated subjective outcome measurement is questionable. Patients had to answer questions concerning the last 5 years, which may have given recall bias. The long-term outcome could be influenced by the alternative treatments that the patients received after completion of the heel-drop programme. However, of the patients who did not receive alternative treatments, $56.7 \%$ were completely pain-free after 5 years. Robinson et a ${ }^{17}$ recommended to use the VISA-A score in a homogenous group of athletes. Especially at long-term follow-up, the VISA-A score questionnaire can be subject to other influences than solely the symptoms of the Achilles tendinopathy. Factors like general ageing and new comorbidity may have influenced the outcome in this study. Finally, the ultrasonographic examinations at baseline and at follow-up were performed by different observers. However, in an earlier study, an excellent interobserver reliability of grading the neovascularisation was found. ${ }^{32}$

\section{What this study adds}

Patients with chronic Achilles tendinopathy treated with Alfredson's heel-drop exercise programme continue to improve, as measured by the VISA-A score, up to 5 years after completion of the programme.

\section{CONCLUSION}

This is the first study evaluating the 5-year results of the widely used heel-drop exercise programme as described by Alfredson et al with the validated VISA-A score. At 5 -year follow-up, the VISA-A score increased significantly.

After the 3-month eccentric training programme, almost half of the patients had received other therapies. Although improvement of symptoms can be expected at long term, mild pain may remain.

Contributors Study concept and design: RJdV, JLT, AW, JANV. Acquisition of data: AvdP. Analysis and interpretation of data: AvdP, SdJ, RJdV, JLT, HJLvdH, JANV. Drafting of the manuscript: AvdP, SdJ, JLT, RJdV. Critical revision of the manuscript for important intellectual content: AvdP, SdJ, JLT, RJdV, AW, HJLvdH, JANV. Statistical analysis: AvdP, HJLvdH. Administrative, technical or material support: AvdP, SdJ, RJdV, JLT, AW. Study supervision: RJdV, JLT, HJLvdH, JANV.

Acknowledgements The authors thank Esaote Piemedical for permission to use the power Doppler machine. The Rheumatology Department (The Hague Medical Centre Antoniushove) and T van der Werf are also thanked for help and advice during this study.

\section{Competing interests None.}

Ethics approval The study was approved by the Medical Ethics Committee of the Hospital.

Provenance and peer review Not commissioned; externally peer reviewed.

\section{REFERENCES}

1. Kingma JJ, de Knikker R, Wittink HM, et al. Eccentric overload training in patients with chronic Achilles tendinopathy: a systematic review. Br J Sports Med 2007:41:e3.

2. Kujala UM, Sarna S, Kaprio J. Cumulative incidence of achilles tendon rupture and tendinopathy in male former elite athletes. Clin J Sport Med 2005;15:133-5.

3. Magnussen RA, Dunn WR, Thomson AB. Nonoperative treatment of midportion Achilles tendinopathy: a systematic review. Clin J Sport Med 2009;19:54-64.

4. Woodley BL, Newsham-West RJ, Baxter GD. Chronic tendinopathy: effectiveness of eccentric exercise. Br J Sports Med 2007;41:188-98; discussion 199 .

5. Allison GT, Purdam C. Eccentric loading for Achilles tendinopathy strengthening or stretching? Br J Sports Med 2009;43:276-9.

6. Meyer A, Tumilty S, Baxter GD. Eccentric exercise protocols for chronic noninsertional Achilles tendinopathy: how much is enough? Scand J Med Sci Sports 2009;19:609-15.

7. Khan KM, Scott A. Mechanotherapy: how physical therapists' prescription of exercise promotes tissue repair. Br J Sports Med 2009;43:247-52.

8. Alfredson H, Pietilä T, Jonsson P, et al. Heavy-load eccentric calf muscle training for the treatment of chronic Achilles tendinosis. Am J Sports Med 1998;26:360-6.

9. Fahlström M, Jonsson P, Lorentzon R, et al. Chronic Achilles tendon pain treated with eccentric calf-muscle training. Knee Surg Sports Traumatol Arthrosc 2003;11:327-33

10. Mafi N, Lorentzon R, Alfredson H. Superior short-term results with eccentric calf muscle training compared to concentric training in a randomized prospective multicenter study on patients with chronic Achilles tendinosis. Knee Surg Sports Traumatol Arthrosc 2001;9:42-7.

11. Roos EM, Engström M, Lagerquist A, et al. Clinical improvement after 6 weeks of eccentric exercise in patients with mid-portion Achilles tendinopathy - a randomized trial with 1-year follow-up. Scand J Med Sci Sports 2004;14:286-95.

12. Silbernagel KG, Thomeé $R$, Thomeé $P$, et al. Eccentric overload training for patients with chronic Achilles tendon pain - a randomised controlled study with reliability testing of the evaluation methods. Scand J Med Sci Sports 2001;11:197-206.

13. de Vos RJ, Weir A, Visser RJ, et al. The additional value of a night splint to eccentric exercises in chronic midportion Achilles tendinopathy: a randomised controlled trial. Br J Sports Med 2007;41:e5. 
14. Gärdin A, Movin T, Svensson L, et al. The long-term clinical and MRI results following eccentric calf muscle training in chronic Achilles tendinosis. Skeletal Radiol 2010;39:435-42.

15. Ohberg L, Lorentzon R, Alfredson H. Eccentric training in patients with chronic Achilles tendinosis: normalised tendon structure and decreased thickness at follow up. Br J Sports Med 2004;38:8-11; discussion 11.

16. Silbernagel KG, Brorsson A, Lundberg M. The majority of patients with Achilles tendinopathy recover fully when treated with exercise alone: a 5-year follow-up. Am J Sports Med 2011;39:607-13.

17. Robinson JM, Cook JL, Purdam C, et al. The VISA-A questionnaire: a valid and reliable index of the clinical severity of Achilles tendinopathy. Br J Sports Med 2001;35:335-41.

18. de Jonge S, de Vos RJ, Van Schie HT, et al. One-year follow-up of a randomised controlled trial on added splinting to eccentric exercises in chronic midportion Achilles tendinopathy. Br J Sports Med 2010;44:673-7.

19. Yang X, Pugh ND, Coleman DP, et al. Are Doppler studies a useful method of assessing neovascularization in human Achilles tendinopathy? A systematic review and suggestions for optimizing machine settings. J Med Eng Technol 2010;35:365-72.

20. Maffulli N, Longo UG, Loppini $\mathrm{M}$, et al. New options in the management of tendinopathy. Open Access J Sports Med 2010:1:29-37.

21. Maffulli N, Walley G, Sayana MK, et al. Eccentric calf muscle training in athletic patients with Achilles tendinopathy. Disabil Rehabil 2008; 30:1677-84.

22. Sayana MK, Maffulli N. Eccentric calf muscle training in non-athletic patients with Achilles tendinopathy. J Sci Med Sport 2007:10:52-8.

23. September AV, Cook J, Handley CJ, et al. Variants within the COL5A1 gene are associated with Achilles tendinopathy in two populations. Br J Sports Med 2009:43:357-65.
24. Divani K, Chan 0, Padhiar N, et al. Site of maximum neovascularisation correlates with the site of pain in recalcitrant mid-tendon Achilles tendinopathy. Man Ther 2010;15:463-8.

25. Ohberg L, Alfredson H. Effects on neovascularisation behind the good results with eccentric training in chronic mid-portion Achilles tendinosis? Knee Surg Sports Traumatol Arthrosc 2004;12:465-70.

26. Reiter M, Ulreich N, Dirisamer A, et al. Colour and power Doppler sonography in symptomatic Achilles tendon disease. Int J Sports Med 2004;25:301-5.

27. van Snellenberg W, Wiley JP, Brunet G. Achilles tendon pain intensity and level of neovascularization in athletes as determined by color Doppler ultrasound. Scand J Med Sci Sports 2007;17:530-4.

28. Archambault JM, Wiley JP, Bray RC, et al. Can sonography predict the outcome in patients with achillodynia? J Clin Ultrasound 1998;26:335-9.

29. Richards PJ, McCall IW, Day C, et al. Longitudinal microvascularity in Achilles tendinopathy (power Doppler ultrasound, magnetic resonance imaging timeintensity curves and the Victorian Institute of Sport Assessment-Achilles questionnaire): a pilot study. Skeletal Radiol 2010;39:509-21.

30. Richards PJ, Win T, Jones PW. The distribution of microvascular response in Achilles tendonopathy assessed by colour and power Doppler. Skeletal Radiol 2005; 34:336-42.

31. Boesen MI, Koenig MJ, Torp-Pedersen S, et al. Tendinopathy and Doppler activity: the vascular response of the Achilles tendon to exercise. Scand J Med Sci Sports 2006;16:463-9.

32. Sengkerij PM, de Vos RJ, Weir A, et al. Interobserver reliability of neovascularization score using power Doppler ultrasonography in midportion achilles tendinopathy. Am J Sports Med 2009;37:1627-31. 